\title{
Resolving Loads with Positive Interior Stresses ${ }^{\star}$
}

\author{
Günter Rote ${ }^{1}$ and André Schulz ${ }^{2 \star \star}$ \\ 1 Institut für Informatik, Freie Universität Berlin, Germany, rote@inf.fu-berlin.de \\ 2 Department of Computer Science, Smith College, USA, aschulz@email.smith.edu
}

\begin{abstract}
We consider the pair $\left(\mathbf{p}_{i}, \mathbf{f}_{i}\right)$ as a force with two-dimensional direction vector $\mathbf{f}_{i}$ applied at the point $\mathbf{p}_{i}$ in the plane. For a given set of forces we ask for a non-crossing geometric graph on the points $\mathbf{p}_{i}$ that has the following property: There exists a weight assignment to the edges of the graph, such that for every $\mathbf{p}_{i}$ the sum of the weighted edges (seen as vectors) around $\mathbf{p}_{i}$ yields $-\mathbf{f}_{i}$. As additional constraint we restrict ourselves to weights that are non-negative on every edge that is not on the convex hull of the point set. We show that (under a generic assumption) for any reasonable set of forces there is exactly one pointed pseudo-triangulation that fulfils the desired properties. Our results will be obtained by linear programming duality over the PPT-polytope. For the case where the forces appear only at convex hull vertices we show that the pseudo-triangulation that resolves the load can be computed as weighted Delaunay triangulation. Our observations lead to a new characterization of pointed pseudo-triangulations, structures that have been proven to be extremely useful in the design and analysis of efficient geometric algorithms.

As an application, we discuss how to compute the maximal locally convex function for a polygon whose corners lie on its convex hull.
\end{abstract}

\section{Introduction}

Let $P=\left\{\mathbf{p}_{1}, \ldots, \mathbf{p}_{n}\right\}$ be a set of distinct points in the plane in general position and let $F=\left\{\mathbf{f}_{1}, \ldots, \mathbf{f}_{n}\right\}$ denote a set of two-dimensional vectors. We think of the pair $\left(\mathbf{p}_{i}, \mathbf{f}_{i}\right)$ as a force in direction $\mathbf{f}_{i}$ that is applied at the point $\mathbf{p}_{i}$. The set of pairs $L=\left\{\left(\mathbf{p}_{1}, \mathbf{f}_{1}\right), \ldots,\left(\mathbf{p}_{n}, \mathbf{f}_{n}\right)\right\}$ is called a load. The objects we study in this paper are geometric graphs $G=(P, E)$ with point set $P$ and edge set $E$. An edge of $E$ that is not part of the convex hull of $P$ is considered as interior edge.

A stress is a (symmetric) assignment of scalars to the edges $E$. Throughout the paper we denote a stress of $G$ with $\omega: P \rightarrow \mathbb{R}$. We say that a graph resolves a load $L$ with stress $\omega$, if

$$
\forall \mathbf{p}_{i}: \sum_{(i, j) \in E} \omega_{i j}\left(\mathbf{p}_{i}-\mathbf{p}_{j}\right)=-\mathbf{f}_{i} .
$$

We can interpret condition (1) as follows: Consider the edges meeting in a point as vectors; the sum of these vectors (weighted by $\omega$ ) cancels the vector $\mathbf{f}_{i}$. If there exists some stress $\omega$ for which $G$ resolves the load $L$ we say that $G$ resolves $L$. Furthermore, if there exists a stress $\omega$, positive or zero on every interior edge, we say that $G$ resolves $L$ with positive interior stress. Stressed graphs have a

\footnotetext{
* Author-created version. (C)Springer-Verlag Berlin Heidelberg 2009. To appear.

** Funded by the German Research Foundation (DFG) under grant 2458/1-1.
} 
physical interpretation. Their edges can be considered as a system of springs. Due to Hooke's law the force induced by a spring is proportional to its length. Hence the values $\omega_{i j}$ are the spring constants from this point of view. A negative spring constant models a rubber band - thus by considering only positive interior stresses we restrict ourselves to (expansive) springs.

In this paper we study the problem how to find a graph that resolves a given load with positive interior stress. Figure 1(a) shows a small introductory example of a problem instance. A possible solution with the corresponding stress is depicted in Figure 1(b).

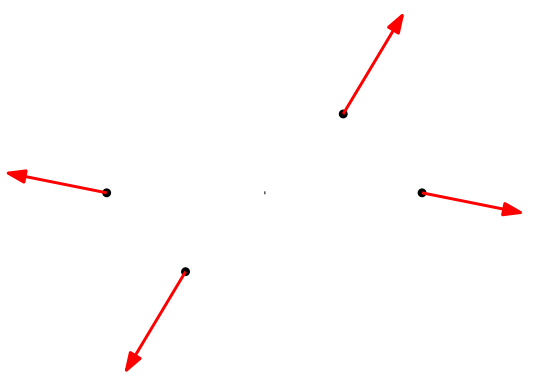

(a)

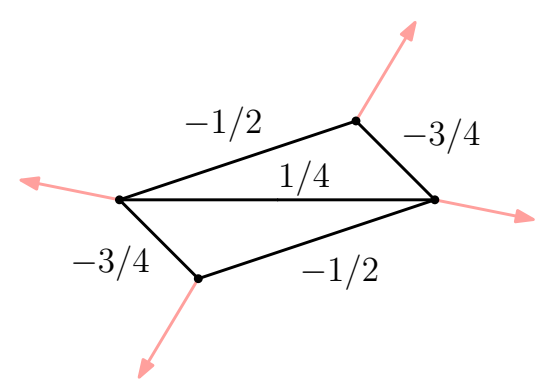

(b)

Fig. 1. A small example.

Not all loads can be resolved by a graph. In particular, a load has to contribute neither a linear momentum (that is $\sum_{i} \mathbf{f}_{i}=\mathbf{0}$ ) nor an angular momentum (that is $\left.\sum_{i}\left\langle\mathbf{f}_{i}, \mathbf{p}_{i}^{\perp}\right\rangle=0\right)$. The vector $\mathbf{p}^{\perp}:=(y,-x)^{T}$ denotes the rotation of $\mathbf{p}=(x, y)^{T}$ by 90 degrees around the origin. To see this, observe the following: Every geometric graph with fixed stress can resolve exactly one load. If the graph consists of a single edge $(i, j)$ it can resolve the load given by $\mathbf{f}_{i}=\omega_{i j}\left(\mathbf{p}_{j}-\mathbf{p}_{i}\right)$ and $\mathbf{f}_{j}=\omega_{i j}\left(\mathbf{p}_{i}-\mathbf{p}_{j}\right)$. We notice that $\mathbf{f}_{i}$ and $\mathbf{f}_{j}$ sum up to $\mathbf{0}$ and that $\left\langle\mathbf{f}_{i}, \mathbf{p}_{i}^{\perp}\right\rangle+\left\langle\mathbf{f}_{j}, \mathbf{p}_{j}^{\perp}\right\rangle=0$ holds. A load resolved by a stressed graph is the composition of these "atomic" forces induced by single edges. Since the total linear (angular) momentum is the sum of the linear (angular) momenta of the atomic forces we cannot resolve a load with non-vanishing linear or angular momentum. In the following we consider only loads without linear and angular momentum. If we want to emphasize the absence of a momentum we use the term moment-free for loads.

If a graph can resolve every moment-free load it is called statically rigid. It is well known that static rigidity is the dual concept to infinitesimal rigidity. In other words, a graph is statically rigid if and only if it is infinitesimally rigid. This observation was already noticed by Föppl [14] and Henneberg [16, page 456]. In $2 \mathrm{~d}$ (generically) minimally rigid graphs can be characterized by a combinatorial counting argument which was given by Laman [18]. These graphs are called Laman graphs and are (statically) rigid in almost every realization in the plane. The question we study in this paper is from a slightly different nature - we look for graphs that can resolve only a fixed load. On the other hand, we have as additional constraint that the stress on interior edges has to be non-negative. 
The load given by $\mathbf{f}_{i}=\mathbf{0}$ for all $\mathbf{p}_{i}$ is called zero load. A stress that resolves this load on $G$ is called equilibrium stress for $G$. Graphs with equilibrium stresses have several nice properties. An old results that goes back to James Clerk Maxwell states that the equilibrium stresses of a planar graph are in one-to-one correspondence with its spatial liftings [21]. Furthermore, the sign of the stress on an edge indicates the curvature along this edge in the lifting. This observation is known as the Maxwell-Cremona correspondence.

Considering positive interior stresses only is also motivated by the construction of a discrete Laplace-Beltrami operator [32]. The Laplace-Beltrami Operator has applications in surface parameterization [13], symmetry detection [22], mesh filtering [12], and physical simulation [31], just to mention a few. There exist different versions of the discrete Laplace-Beltrami operator, but none can guarantee all properties of the continuous equivalent [32]. In particular, a discrete LaplaceBeltrami operator for a triangulated surface mesh should be modeled by an equilibrium stress (with every vertex incident to at least one non-zero stress) whenever the surface lies in the plane. On the other hand the edge weights should be nonnegative to guarantee the maximum principle, which is a natural property for the classical Laplace-Beltrami operator and which should also hold for the discrete version [5]. As mentioned in [32], the existence of non-regular triangulations makes it impossible to construct a "perfect" discrete Laplacian. However, it is open how to fix this inconvenience by local adjustments to the original mesh. A better understanding how positive equilibrium stresses behave could lead to a solution for this problem.

We restrict ourselves to a special class of graphs that might resolve a given load with a positive interior stress. These are the pointed pseudo-triangulations. A pseudo-triangulation of $P$ is a partition of the convex hull of $P$ into polygons with three corners. such that every $\mathbf{p}_{i}$ is part of some polygon. A corner is a vertex of a polygon with interior angle smaller than $\pi$. If every point is incident to an angle greater than $\pi$ the pseudo-triangulation is called pointed. A pseudo-triangulations can resolve any moment-free load with positive and negative stresses (Streinu [30]). Pseudo-triangulations are related to maximal locally convex functions. This relationship was observed by Aichholzer et al. [2] and was further extended by Aurenhammer and Krasser [3]. Similar to triangulations pointed pseudo-triangulations appear as geometric data structures and they are used to prove the correctness and efficiency of algorithms. They find applications in ray shooting [6], motion planning [4], and art gallery type problems [29]. This list is far from complete, for a comprehensive discussion on pseudo-triangulations we direct the reader to the survey by Rote, Santos and Streinu [26].

The reasons why we focus on pointed pseudo-triangulations are the following. Since they can resolve loads with general stresses, they seem to be powerful enough. On the other hand, every vertex which is not pointed is in some sense "overconstrained" to resolve the force (with positive stresses) at this point, because the effect of at least one stressed edge can be expressed by adjusting the stresses on two other edges. Moreover, pseudo-triangulations are non-crossing geometric graphs and therefore easy to understand for the viewer.

Results: As our main result we show that there exists for every moment-free load $L$ a pointed pseudo-triangulation that can resolve $L$ with positive interior stress. 
Moreover, up to degenerate situations, this pointed pseudo-triangulation is unique. We extend these results to a constrained version of the original problem. This means that even if we restrict a certain set of edges to appear in the solution, we can find a pointed pseudo-triangulation that contains this set and resolves $L$ with positive stress on the unconstrained interior edges. This is true for any constraints that allow the completion to some pointed pseudo-triangulation. As in the original setting the obtained solution is unique for almost every load. The constrained and the unconstrained problem can be solved by linear programming. For the special situation when all forces appear only at convex hull vertices we provide an algorithm that computes the load resolving pointed pseudo-triangulation without linear programming.

As application we show how we can compute pointed pseudo-triangulations that refer to maximal locally convex functions, for polygons whose corners lie on its convex hull. Our approach combines the load-resolving method with the Maxwell-Cremona correspondence.

\section{Resolving Loads with Pointed Pseudo-Triangulations}

\subsection{General Solution}

Let us start with some preliminary observations about pointed pseudotriangulations. In the following we use pointed pseudo-triangulations of point sets and (later in Section 3) also of simple polygons.

Definition 1. A pointed pseudo-triangulation of a point set $P$ is the partition of the convex hull of $P$ into polygons with three corners, such that every vertex in $P$ is incident to an angle greater than $\pi$.

$A$ pointed pseudo-triangulation of a polygon $\mathcal{P}$ is the partition of $\mathcal{P}$ into polygons with three corners, such that every vertex of $\mathcal{P}$ is incident to an angle greater than $\pi$.

There exists a high-dimensional polytope whose corners correspond to the pointed pseudo-triangulations a point set can have [25]. This polytope is called PPTpolytope and it is based on the fact that every pointed pseudo-triangulation has an expansive infinitesimal motion, if one removes a convex hull edge (see Streinu [30]). The infinitesimal velocities $\mathbf{v}_{1}, \ldots, \mathbf{v}_{n}$ (each $\mathbf{v}_{i}$ is a two-dimensional vector) act as unknowns in the description of the polytope:

$$
\begin{aligned}
\left\langle\mathbf{v}_{i}-\mathbf{v}_{j}, \mathbf{p}_{i}-\mathbf{p}_{j}\right\rangle & \geq\left\langle\mathbf{p}_{i}, \mathbf{p}_{j}^{\perp}\right\rangle^{2} \quad \forall i, j \leq n, \\
\left\langle\mathbf{v}_{i}-\mathbf{v}_{j}, \mathbf{p}_{i}-\mathbf{p}_{j}\right\rangle & =\left\langle\mathbf{p}_{i}, \mathbf{p}_{j}^{\perp}\right\rangle^{2} \quad \text { for }(i, j) \in \operatorname{conv}(P), \\
\sum_{i=1}^{n} \mathbf{v}_{i} & =\mathbf{0}, \\
\sum_{i=1}^{n}\left\langle\mathbf{v}_{i}, \mathbf{p}_{i}^{\perp}\right\rangle & =0 .
\end{aligned}
$$

The PPT-polytope is a simple polytope with dimension $2 n-3$. Hence, in each of its vertices $2 n-3$ inequalities are tight (including the equations of the convex hull edges). The pointed pseudo-triangulation that is associated with a specific vertex of the PPT-polytope is given by the edges induced by its tight inequalities. 
We study the minimization of the function

$$
\sum_{i=1}^{n}\left\langle\mathbf{v}_{i},-\mathbf{f}_{i}\right\rangle
$$

over the PPT-polytope given by $(2-4)$ (in the following considered as primal program). As we will see later our choice of the objective function (5) leads to a solution that is capable to resolve the load $L$.

The constraints of the corresponding dual program have the following form:

$$
1 \leq i \leq n: \sum_{j=1}^{n} u_{i j}\left(\mathbf{p}_{i}-\mathbf{p}_{j}\right)+\mathbf{t}+r \mathbf{p}_{i}^{\perp}=-\mathbf{f}_{i} .
$$

The variables $\mathbf{t}$ and $r$ correspond to the equations (3) and (4). For every possible interior edge we obtain by LP duality the dual constraint $u_{i j} \geq 0$. By complementary slackness we deduce that if a constraint is not tight in the primal solution (there is no edge defined by this inequality), then the corresponding dual variable $u_{i j}$ is zero in the dual solution. Thus, a (non-zero) $u_{i j}$ appears only on the edges of the primal solution.

We observe that the dual variables $\mathbf{t}$ and $r$ that come from the conditions (3) and (4) are the only difference between (6) and (1). Fortunately, we can show that under our assumptions the variables $\mathbf{t}$ and $r$ can only be zero.

Lemma 1. If the load in the primal program is moment-free we have for the dual variables $\mathbf{t}=\mathbf{0}$ and $r=0$.

Proof. Equation (6) denotes $n$ restrictions of the dual program. Adding up all these equations cancels the $u_{i j}$ variables and gives

$$
n \mathbf{t}+r\left(\sum_{i=1}^{n} \mathbf{p}_{i}\right)^{\perp}=-\sum_{i=1}^{n} \mathbf{f}_{i}
$$

Because the objective function is moment-free the last equation equals zero. Now, we take the scalar product of both sides of equation (6) with $\mathbf{p}_{i}^{\perp}$. This gives $n$ equations of the form

$$
-\sum_{j=1}^{n} u_{i j}\left\langle\mathbf{p}_{i}^{\perp}, \mathbf{p}_{j}\right\rangle+\left\langle\mathbf{p}_{i}^{\perp}, \mathbf{t}\right\rangle+r\left\|\mathbf{p}_{i}\right\|^{2}=-\left\langle\mathbf{p}_{i}^{\perp}, \mathbf{f}_{i}\right\rangle .
$$

If we sum up all these equations, the $u_{i j}$ variables cancel, since $\left\langle\mathbf{p}_{i}^{\perp}, \mathbf{p}_{j}\right\rangle=\left\langle\mathbf{p}_{i}^{\perp \perp}, \mathbf{p}_{j}^{\perp}\right\rangle=$ $\left\langle-\mathbf{p}_{i}, \mathbf{p}_{j}^{\perp}\right\rangle=-\left\langle\mathbf{p}_{j}^{\perp}, \mathbf{p}_{i}\right\rangle$. We obtain

$$
\left\langle\left(\sum_{i=1}^{n} \mathbf{p}_{i}\right)^{\perp}, \mathbf{t}\right\rangle+r \sum_{i=1}^{n}\left\|\mathbf{p}_{i}\right\|^{2}=-\sum_{i=1}^{n}\left\langle\mathbf{p}_{i}^{\perp}, \mathbf{f}_{i}\right\rangle .
$$

Again this equation is zero because the objective function is moment-free.

The variables $\mathbf{t}$ and $r$ can be computed by solving a homogeneous linear equation system given by the three equations from (7) and (9). It remains to show that 
$\mathbf{t}=\mathbf{0}$ and $r=0$ is the only solution of this system. We can rephrase (7) to express $\mathbf{t}$ as

$$
\mathbf{t}=-\frac{r}{n}\left(\sum_{i=1}^{n} \mathbf{p}_{i}\right)^{\perp} .
$$

If $r=0$ then $\mathbf{t}=\mathbf{0}$ and we get the trivial solution. Therefore, let us assume that $r$ is nonzero. We plug (10) into equation (9) and obtain

$$
-\frac{r}{n}\left\langle\left(\sum_{i=1}^{n} \mathbf{p}_{i}\right)^{\perp},\left(\sum_{i=1}^{n} \mathbf{p}_{i}\right)^{\perp}\right\rangle+r \sum_{i=1}^{n}\left\|\mathbf{p}_{i}\right\|^{2}=0 .
$$

Further simplifications give

$$
\sum_{i=1}^{n}\left\|\mathbf{p}_{i}\right\|^{2}-\frac{1}{n}\left\|\sum_{i=1}^{n} \mathbf{p}_{i}\right\|^{2}=0 .
$$

Let $\overline{\mathbf{p}}:=\frac{1}{n} \sum_{i=1}^{n} \mathbf{p}_{i}$ denote the center of gravity of $P$. We deduce

$$
\left\|\mathbf{p}_{i}\right\|^{2}=\left\|\overline{\mathbf{p}}+\mathbf{p}_{i}-\overline{\mathbf{p}}\right\|^{2}=\|\overline{\mathbf{p}}\|^{2}+2\left\langle\overline{\mathbf{p}}, \mathbf{p}_{i}-\overline{\mathbf{p}}\right\rangle+\left\|\mathbf{p}_{i}-\overline{\mathbf{p}}\right\|^{2} .
$$

Plugging this equivalence into equation (11) leads to

$$
\begin{aligned}
\sum_{i=1}^{n}\left(\|\overline{\mathbf{p}}\|^{2}+2\left\langle\overline{\mathbf{p}}, \mathbf{p}_{i}-\overline{\mathbf{p}}\right\rangle+\left\|\mathbf{p}_{i}-\overline{\mathbf{p}}\right\|^{2}\right)-n\|\overline{\mathbf{p}}\|^{2} & =n\|\overline{\mathbf{p}}\|^{2}+\sum_{i=1}^{n}\left\|\mathbf{p}_{i}-\overline{\mathbf{p}}\right\|^{2}-n\|\overline{\mathbf{p}}\|^{2} \\
& =\sum_{i=1}^{n}\left\|\mathbf{p}_{i}-\overline{\mathbf{p}}\right\|^{2}
\end{aligned}
$$

We observe that the last expression is strictly positive as long as not all $\mathbf{p}_{i}$ are the same, which is not allowed in our case. Hence, $\mathbf{t}=\mathbf{0}$ and $r=0$ is the only solution of the homogeneous system and the lemma follows.

As consequence of Lemma 1 the dual variables $u_{i j}$ define a stress that resolves the load $L$ and is positive on every interior edge. Hence, the solution of the primal program computes a graph with the desired properties. Notice that almost every objective function has a unique solution, and thus there is for almost every load exactly one pointed pseudo-triangulation that resolves it with positive interior stress.

Theorem 1 (Main Theorem). For every moment-free load $L$ there exists a pointed pseudo-triangulation that resolves $L$ with positive interior stress. Up to degenerate situations this pointed pseudo-triangulation is unique and it is the solution of the linear program (2-5).

Algorithmically, the computation of the desired pointed pseudo-triangulation boils down to solving a linear program with $2 n$ variables, whose length is in $O\left(n^{2}\right)$. Various methods and tools are applicable to solve LP programs. The interior point method of Karmarkar [17] runs in $O\left(n^{3.5} K\right)$, where $K$ is the number of input bits. 


\subsection{The Constrained Problem}

We are looking now for a pointed pseudo-triangulation that resolves a load $L$ with positive interior stress and that contains a prescribed set of edges $E_{c}$. Notice that a set of non-crossing edges that leaves an angle greater than $\pi$ at every vertex can always be completed to a pointed pseudo-triangulation [30]. Let us assume that $E_{c}$ allows the completion to a pointed pseudo-triangulation on $P$.

The solution in this constrained setting can be computed with the same method we used for the general case. Again, we use a linear program to compute the pointed pseudo-triangulation. But this time we optimize only over a facet of the PPT-polytope. This facet can be obtained by turning all inequalities of (2) that refer to edges in $E_{c}$ into equations:

$$
\left\langle\mathbf{v}_{i}-\mathbf{v}_{j}, \mathbf{p}_{i}-\mathbf{p}_{j}\right\rangle=\left\langle\mathbf{p}_{i}, \mathbf{p}_{j}^{\perp}\right\rangle^{2}, \quad \text { for }(i, j) \in E_{c} .
$$

The facet of the PPT-polytope is simple and, more important, it is not empty.

Forcing edges to appear in the graph has the following consequences for our LP approach: The inequalities of $E_{c}$ are now equations. Thus, we have no information about the sign of the corresponding dual variables $u_{i j}$ anymore. On the other hand the dual restrictions (6) are not affected. We have still $u_{i j} \geq 0$ for every $u_{i j}$ that does not refer to an edge in $E_{c}$. Notice that Lemma 1 can be applied in this constrained setting without modifications. As a consequence we can deduce:

Theorem 2. Let $L=P \times F$ be a moment free load and $E_{c}$ be a set of edges that allows the completion to a pointed pseudo-triangulation on $P$. There exists a pointed pseudo-triangulation that contains $E_{c}$ and resolves $L$ with a stress that is positive on every interior edge that is not in $E_{c}$. Up to degenerate situations this pointed pseudo-triangulation is unique and it is the solution of the linear program given by (2-5) and (12).

\subsection{No Interior Forces}

Let us assume for this section that $\mathbf{f}_{i}=\mathbf{0}$ for every vertex that is not on the convex hull of $P$. Under this assumption, we can find the pseudo-triangulation that resolves $L$ by geometric methods, without solving a linear program. If we have no edge constraints, all interior points can simply be ignored: in this setting, we are looking for a triangulation that resolves forces whose points of application are in convex position.

Let us assume that the facial structure of a planar graph $G$ is given by some combinatorial embedding (a planar map), and in addition, the vertices of $G$ are drawn in the plane and the edges are realized as straight lines (a geometric graph). Note that the drawing in the plane can have crossings, and is not necessarily related to the facial structure. A height assignment $h: P \rightarrow \mathbb{R}$ is a lifting of a graph if all vertices that belong to a face lie on a common plane in $\mathbb{R}^{3}$ when giving $\mathbf{p}_{i}$ the additional coordinate $z_{i}=h\left(\mathbf{p}_{i}\right)$. The relation between liftings of a planar graph that is drawn as a geometric graph in the plane and its equilibrium stresses is expressed by the Maxwell-Cremona correspondence, which goes back to James Clerk Maxwell [21]. 
Theorem 3 (Maxwell-Cremona correspondence). For a planar 2-connected graph $G$ drawn in the plane, with a designated face $\hat{f}$, there is a one-to-one correspondence between

1. the liftings of $G$ with $\hat{f}$ in the $x y$-plane, and

2. the equilibrium stresses on $G$.

When we apply this theorem in this paper, the lifted surface is usually a polyhedral surface that consists of an "upper surface" and a "lower surface" that are glued together at their common boundary. Each part, when individually projected to the plane, will yield a planar drawing without crossings, but the overlay of the two parts will in general have crossings. It is possible that the lower and the upper surface intersect each other; this is no problem. When $G$, or a part of $G$, is drawn without crossings and the faces of $G$ are the faces of this drawing, an edge with a positive stress lifts to a convex edge and an edge with a negative stress a concave edge.

The complete proof of the Maxwell-Cremona correspondence is due to Whiteley [33] (see also [11]). A more constructive proof is due to Richter-Gebert [24]. The Maxwell-Cremona correspondence finds application in polygon unfolding [9] and grid embeddings of 3-polytopes [23]. Once we have a equilibrium stress, the computation of the lifting is easy and can be computed face by face, starting with $\hat{f}$. For the detailed rules how to compute the lifting we refer to Richter-Gebert's book [24].

We first discuss the unconstrained load resolving problem. We look for a graph that resolves $L_{C}:=\left\{\left(\mathbf{p}_{i}, \mathbf{f}_{i}\right) \mid \mathbf{p}_{i}\right.$ lies on the convex hull $\}$. But this time negative stresses on interior edges are allowed. It is known that every triangulation can resolve $L_{C}$ since it is a so called Laman graph and hence statically rigid [14]. Let us pick an arbitrary triangulation $T$ of the convex hull. We compute the stress that resolves $L_{C}$ on $T$ and multiply all stresses by -1 . This gives a stressed graph that "produces" the load $L_{C}$. The stressed triangulation $T$ combined with the triangulation $T^{\prime}$ that resolves $L_{C}$ with positive interior stresses yields a planar graph with equilibrium stress. Thus, by Maxwell-Cremona there is a lifting of this composition. From this lifting we know the heights of the lifted points, because every point lies on a face of $T$ and we can compute the lifting partially for the faces of $T$, when we choose $\hat{f}$ as face of $T$. Since the interior edges of $T^{\prime}$ are restricted to have a positive stress their curvature in the lifting of $T \cup T^{\prime}$ yields a convex bending. Therefore, the combinatorial structure of $T^{\prime}$ coincides with the weighted Delaunay triangulation of $\operatorname{conv}(P)$. The Delaunay-weights are given by $\left\|p_{i}\right\|^{2}-z_{i}$ for every convex hull vertex $\mathbf{p}_{i}$. The weighted Delaunay triangulation for convex point sets can be computed in linear time [1].

The stresses of $T$ can be computed with help of an ear decomposition of $T$. Let $\mathbf{p}_{c}$ be the corner of an ear of $T$. The force $\left(\mathbf{p}_{c}, \mathbf{f}_{c}\right)$ can be canceled by the two boundary edges of $\mathbf{p}_{c}$ by a unique stress which can be easily computed. We can update the forces assigned to the neighbors of $\mathbf{p}_{c}$ by subtracting the vector induced by the (newly) stressed edges incident to $\mathbf{p}_{c}$. Now we can eliminate the ear and continue the ear decomposition in this fashion until we reduced $T$ to a triangle. Notice that by updating the neighboring forces we deal with a moment-free load in every step of the ear decomposition. Thus for the final triangle we have three moment-free forces which can be canceled. The stresses of the final triangle can 
be computed by a small linear system. Since the ear decomposition clearly runs in linear time we can compute the triangulation that resolves $L_{C}$ with positive interior stresses in linear time.

For the constrained setting we can use the same ideas. More precisely, we fix again an arbitrary triangulation $T$ and compute again a stress that resolves $L$ on $T$. The heights of the associated lifting can be computed by the Maxwell-Cremona correspondence. The pointed pseudo-triangulation that resolves $L_{C}$ is characterized by the polyhedral surface that is convex on every line segment inside $\operatorname{conv}(P)$ that doesn't cross a constraint edge. Aichholzer et al. [2] give an algorithm (not based on linear programming) that computes this polyhedral surface. Unfortunately, no practical bounds for the running time of this algorithm are known.

\section{Computing Optimal Pointed Pseudo-Triangulations of Polygons}

Let $\mathcal{P}$ be a polygon with vertex set $P=\left\{\mathbf{p}_{1}, \ldots, \mathbf{p}_{n}\right\}$ and let $P_{c}$ denote the set of corners of $\mathcal{P}$. Furthermore, let $h: P_{c} \rightarrow \mathbb{R}$ be a height assignment for the corners of $\mathcal{P}$. We study the maximal function $f^{*}: \mathcal{P} \rightarrow \mathbb{R}$ that is convex on every line inside $\mathcal{P}$ and that fulfills $h\left(\mathbf{p}_{i}\right)=f^{*}\left(\mathbf{p}_{i}\right)$ for all corners $\mathbf{p}_{i} \in P_{c}$. It was proven by Aichholzer et al. [2] that $f^{*}$ describes a piecewise linear surface, whose non-linearities project down to a pointed pseudo-triangulation $\mathcal{P} \mathcal{T}_{h}$. The framework of [2] is more general and covers also polygons with additional points inside. It can also be used to define optimal non-pointed pseudo-triangulations.

We can compute $\mathcal{P} \mathcal{T}_{h}$ (or $f^{*}$ ) by (i) picking an arbitrary pseudo-triangulation of $\mathcal{P}$ and then (ii) applying a sequence of local adjustments which are called flips. A flip is a transformation of a pseudo-triangulation that exchanges, inserts or removes a single edge and produces a new pseudo-triangulation. There exists a criterion which tells us whether a flip brings us "closer" to $\mathcal{P} \mathcal{T}_{h}$ or not. Thus, we can compute $\mathcal{P} \mathcal{T}_{h}$ by a sequence of such (improving) flips. As a result of [2, Optimality Theorem] we know that the sequence is finite and terminates at $\mathcal{P} \mathcal{T}_{h}$. For a polygon, an improving sequence can have super-polynomial length [28] but we can always find a short sequence of $O\left(n^{2}\right)$ flips [2, Lemma 7.3]. During the flipping process we must keep track of the heights on all vertices of $P \backslash P_{C}$. Only this information allows us to decide if a flip is improving. It was noticed in [15, page 55] that the recomputation of the heights can be expressed by a linear equation system that is based on a planar structure. As mentioned in [7], the Planar Separator Theorem provides a solution in $O(M(\sqrt{n}))$ time in this case, where $M(n)$ is the upper bound for multiplying two $n \times n$ matrices $[19,20]$. The current record for $M(n)$ is $O\left(n^{2.325}\right)$, which is due Coppersmith and Winograd [10]. Thus, a flip can be carried out in $O\left(n^{1.163}\right)$ time, and the whole algorithm takes $O\left(n^{3.163}\right)$ time.

We give an alternative algorithm how to compute $\mathcal{P} \mathcal{T}_{h}$ for polygons whose corners lie on its convex hull. Our approach uses a completely new technique and is based on the observations of the Maxwell-Cremona correspondence, introduced in the previous section. Here, in contrast to the previous section, we use a linear programming approach to solve a geometric problem.

We first give a high-level description of our method. We construct a polyhedron that consists of two shells. Roughly speaking, the upper shell is the (unknown) 
surface given by $f^{*}$-the lower shell is a cone with apex at the origin that spans the lifted corners of the polygon. To glue the two shells together, we have to extend $f^{*}$ to the set $\operatorname{conv}(P) \backslash \mathcal{P}$ (the pockets of $\mathcal{P}$ ). Due to Maxwell-Cremona, the vertical projection of the polyhedron has an equilibrium stress, with positive stressed edges on the interior edges of $\mathcal{P}$. Each shell alone produces a moment-free load that shows up on the convex hull vertices. Since we know the height and the position of the convex hull vertices we know the geometric shape of the lower shell. Hence, we can compute all of its (interior) stresses and therefore the induced moment-free load. The graph that resolves this load with positive interior stresses gives $\mathcal{P} \mathcal{T}_{h}$ with triangulated pockets. Of course we have to enforce the boundary edges of $\mathcal{P}$ to appear in our solution, which can be done by solving the constrained problem as discussed in Section 2.2.

We continue with the detailed construction. Let $[i, j, k, l]$ denote the signed volume of the tetrahedron spanned by the lifted vertices $\mathbf{p}_{i}, \mathbf{p}_{j}, \mathbf{p}_{k}, \mathbf{p}_{l}$ and let $[i, j, k]$ be the signed area of the triangle spanned by the (plane) vertices $\mathbf{p}_{i}, \mathbf{p}_{j}, \mathbf{p}_{k}$. We introduce a new vertex $\mathbf{p}_{0}=(0,0)^{T}$ as the apex of the lower shell. As observed in $[8,28]$, the corresponding stresses on every edge connecting $\mathbf{p}_{0}$ to a corner $\mathbf{p}_{i}$ can be expressed as

$$
\omega_{0 i}:=\frac{[0, h, i, j]}{[0, h, i][0, i, j]},
$$

where $\mathbf{p}_{h}$ is the left neighbor, and $\mathbf{p}_{j}$ is the right neighbor of $\mathbf{p}_{i}$ on the convex hull of $P$. By construction, the stressed edges incident to $\mathbf{p}_{0}$ sum up to $\mathbf{0}$. For every corner $\mathbf{p}_{i}$ we obtain a vector $\mathbf{f}_{i}:=\omega_{0 i}\left(\mathbf{p}_{i}-\mathbf{p}_{0}\right)=\omega_{0 i} \mathbf{p}_{i}$. We use the boundary edges of $\mathcal{P}$ as constraints and compute the pointed pseudo-triangulation that resolves the forces $\mathbf{f}_{i}$.

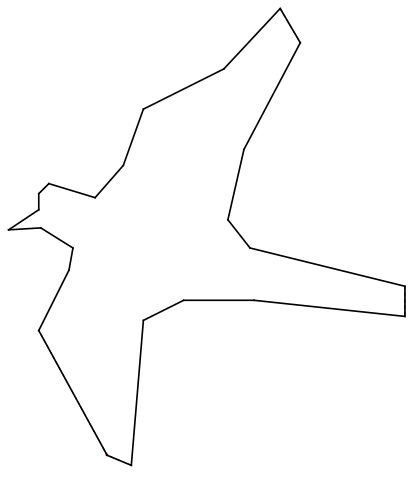

(a)

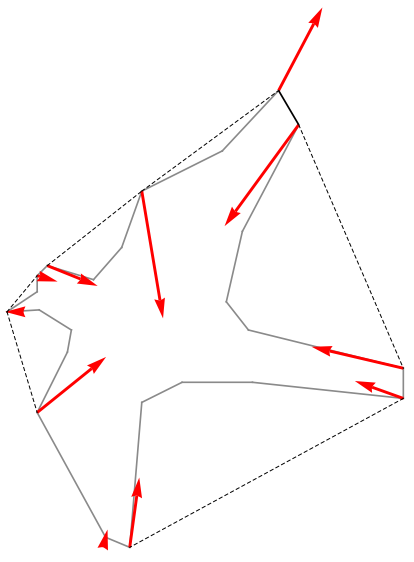

(b)

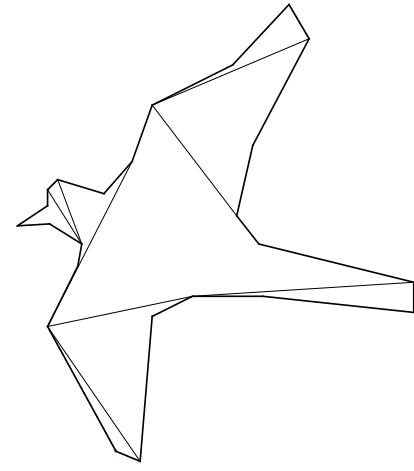

(c)

Fig. 2. A polygon (a), the induced forces that will yield the lifting to the paraboloid (b), and the corresponding pointed Delaunay pseudo-triangulation (c).

We conclude with an example of our method. Figure 2(a) shows a polygon whose corners lie on its convex hull. As height assignment we choose the paraboloid lifting $h\left(\mathbf{p}_{i}\right):=\left\|\mathbf{p}_{i}\right\|^{2}$ — this lifting gives the pointed Delaunay pseudo-triangulation 
of $\mathcal{P}$ (see [27]). The induced lower shell is shown in Figure 3(a) and its induced forces are shown in Figure 2(b). The surface that "fits" into the lower shell and that fulfills all the requirements is depicted in Figure 3(b). It gives the pointed pseudo-triangulation $\mathcal{P} \mathcal{T}_{h}$ of Figure 2(c).

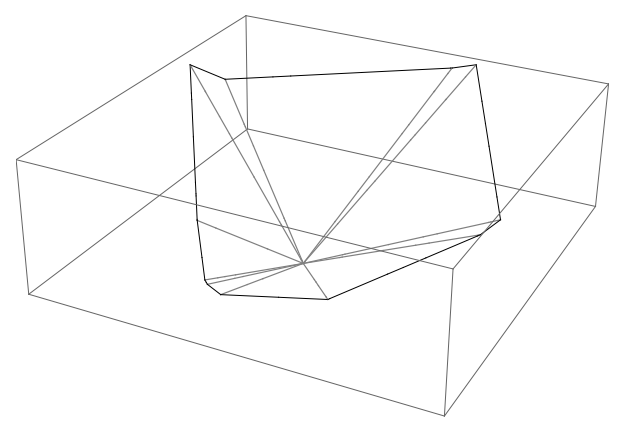

(a)

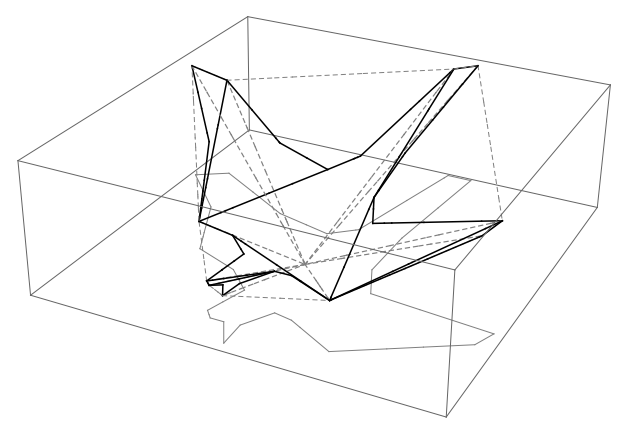

(b)

Fig. 3. Construction of a polyhedron whose upper shell gives $f^{*}$.

Our method extends the result presented in [27] because it allows the computations for a wider class of polygons. The question how to solve the general case with the load resolving method is still open but not out of reach. One has to find a way how to fix the values of the stresses of the edges appearing at the pockets of $\mathcal{P}$ to specify the heights of the corners that are not part of $\operatorname{conv}(P)$. Our algorithm is slower than the execution of the improving flip sequence of [2]. On the other hand our method allows a very simple implementation with the help of an LP-solver.

\section{References}

1. A. Aggarwal, L. J. Guibas, J. Saxe, and P. W. Shor. A linear-time algorithm for computing the Voronoi diagram of a convex polygon. Discrete Comput. Geom., 4(6):591-604, 1989.

2. O. Aichholzer, F. Aurenhammer, P. Brass, and H. Krasser. Pseudo-triangulations from surfaces and a novel type of edge flip. SIAM Journal on Computing, 32:16211653, 2003.

3. F. Aurenhammer and H. Krasser. Pseudo-simplicial complexes from maximal locally convex functions. Discrete $\&$ Computational Geometry, 35(2):201-221, 2006.

4. J. Basch, J. Erickson, L. J. Guibas, J. Hershberger, and L. Zhang. Kinetic collision detection between two simple polygons. Comput. Geom., 27(3):211-235, 2004.

5. A. I. Bobenko and B. Springborn. A discrete Laplace-Beltrami operator for simplicial surfaces. Discrete \& Computational Geometry, 38(4):740-756, 2007.

6. B. Chazelle, H. Edelsbrunner, M. Grigni, L. J. Guibas, J. Hershberger, M. Sharir, and J. Snoeyink. Ray shooting in polygons using geodesic triangulations. Algorithmica, 12:54-68, 1994.

7. M. Chrobak, M. T. Goodrich, and R. Tamassia. Convex drawings of graphs in two and three dimensions (preliminary version). In Proc. 12th Ann. Symposium on Computational Geometry, pages 319-328, 1996. 
8. É. Colin de Verdière, M. Pocchiola, and G. Vegter. Tutte's barycenter method applied to isotopies. Comput. Geom., 26(1):81-97, 2003.

9. R. Connelly, E. D. Demaine, and G. Rote. Straightening polygonal arcs and convexifying polygonal cycles. Discr. Comput. Geometry, 30:205-239, 2003.

10. D. Coppersmith and S. Winograd. Matrix multiplication via arithmetic progressions. J. Symb. Comput., 9(3):251-280, 1990.

11. H. Crapo and W. Whiteley. Plane self stresses and projected polyhedra I: The basic pattern. Structural Topology, 20:55-78, 1993.

12. M. Desbrun, M. Meyer, P. Schröder, and A. H. Barr. Implicit fairing of irregular meshes using diffusion and curvature flow. In SIGGRAPH, pages 317-324, 1999.

13. M. S. Floater and K. Hormann. Surface parameterization: a tutorial and survey. In N. A. Dodgson, M. S. Floater, and M. A. Sabin, editors, Advances in Multiresolution for Geometric Modelling, Mathematics and Visualization, pages 157-186. Springer, Berlin, Heidelberg, 2005.

14. A. Föppl. Theorie des Fachwerks. Verlag Arthur Felix, 1880.

15. R. Haas, D. Orden, G. Rote, F. Santos, B. Servatius, H. Servatius, D. L. Souvaine, I. Streinu, and W. Whiteley. Planar minimally rigid graphs and pseudotriangulations. Comput. Geom., 31(1-2):31-61, 2005.

16. L. Henneberg. Die graphische Statik der starren Systeme. B. G. Teubner, 1911.

17. N. Karmarkar. A new polynomial-time algorithm for linear programming. Combinatorica, 4(4):373-396, 1984.

18. G. Laman. On graphs and rigidity of plane skeletal structures. Journal of Engineering Mathematics, 4(4):331-340, Oct 1970.

19. R. J. Lipton, D. Rose, and R. Tarjan. Generalized nested dissection. SIAM J. Numer. Anal., 16(2):346-358, 1979.

20. R. J. Lipton and R. E. Tarjan. Applications of a planar separator theorem. SIAM J. Comput., 9(3):615-627, 1980.

21. J. C. Maxwell. On reciprocal figures and diagrams of forces. Phil. Mag. Ser., 27:250$261,1864$.

22. M. Ovsjanikov, J. Sun, and L. J. Guibas. Global intrinsic symmetries of shapes. Comput. Graph. Forum, 27(5):1341-1348, 2008.

23. A. Ribó Mor, G. Rote, and A. Schulz. Embedding 3-polytopes on a small grid. In J. Erickson, editor, Proc. 23rd Symposium on Computational Geometry, pages 112118. ACM, 2007.

24. J. Richter-Gebert. Realization Spaces of Polytopes, volume 1643 of Lecture Notes in Mathematics. Springer, 1996.

25. G. Rote, F. Santos, and I. Streinu. Expansive motions and the polytope of pointed pseudo-triangulations. In Discrete and Computational Geometry-The GoodmanPollack Festschrift, volume 25, pages 699-736. Springer, 2003.

26. G. Rote, F. Santos, and I. Streinu. Pseudo-triangulations - a survey. In Surveys on Discrete and Computational Geometry - Twenty Years Later, volume 453 of Contemporary Mathematics, pages 343-410. American Mathematical Society, 2008.

27. G. Rote and A. Schulz. A pointed Delaunay pseudo-triangulation of a simple polygon. In Proceedings of the 21st European Workshop on Computational Geometry, pages 77-80, Eindhoven, 2005.

28. A. Schulz. Lifting planar graphs to realize integral 3-polytopes and topics in pseudotriangulations. PhD thesis, Freie Universität Berlin, 2008.

29. B. Speckmann and C. D. Tóth. Allocating vertex pi-guards in simple polygons via pseudo-triangulations. Discrete $\& 3$ Computational Geometry, 33(2):345-364, 2005.

30. I. Streinu. Pseudo-triangulations, rigidity and motion planning. Discrete $\mathscr{6}$ Computational Geometry, 34(4):587-635, 2005.

31. M. Wardetzky, M. Bergou, D. Harmon, D. Zorin, and E. Grinspun. Discrete quadratic curvature energies. Computer Aided Geometric Design, 24(8-9):499-518, 2007. 
32. M. Wardetzky, S. Mathur, F. Kälberer, and E. Grinspun. Discrete Laplace operators: no free lunch. In SGP 'O7: Proceedings of the Fifth Eurographics Symposium on Geometry Processing, pages 33-37, 2007.

33. W. Whiteley. Motion and stresses of projected polyhedra. Structural Topology, 7:13$38,1982$. 